\title{
Solving BJT Circuits by Nodal Voltage Method with Application of Diakoptical Method
}

\author{
Bohumil Brtník \\ Department of Electrical Engineering \\ University of Pardubice \\ 53002 Pardubice, Czech Republic \\ bohumil.brtnik@upce.cz
}

\begin{abstract}
Bipolar transistor circuits are usually solved by their h-parameters description. However, for circuit solutions are commonly used Nodal Voltage Method based on the $y$-parameters. Therefore it is necessary to convert these h-parameters (hybrid parameters) to y-parameters (admittance parameters) firstly. After then the circuit is solved by hand. But the conversion of parameters is not required when diakoptical method is used. Thus this way of circuit solution is explained in this paper.
\end{abstract}

Keywords- diakoptical method; h-parameters; solving electronic circuits by hand; matrix method.

\section{INTRODUCTION}

The Nodal Voltage Method (NVM) [1] is based on the admittance matrices and the y-parameters. The bipolar junction transistors (BJTs) are usually described by their h-parameters. But conversion of $\mathrm{BJT}$ h-parameters to y-parameters is necessary [2], when the circuit is solved by NVM.

\section{The Derivation OF the Equation SeT}

Illustration circuit is shown on Fig. 1. The figure shows BJT described by its h-parameters (i.e. by $\mathrm{H}$ matrix), which is connected in three nodes into the circuit. But this circuits is described by y-parameters (i.e. by Y matrix).

$$
\begin{aligned}
& I_{1}=g_{1} \cdot V_{1}+I_{B} \\
& I_{2}=g_{2} \cdot V_{2}+I_{C} \\
& I_{3}=g_{3} \cdot V_{3}-I_{B}-I_{C}
\end{aligned}
$$

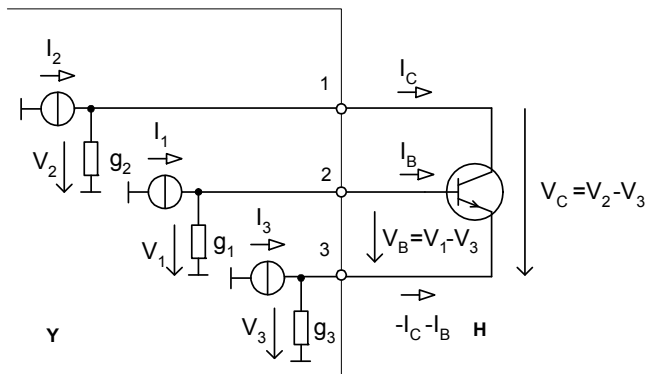

Figure 1. Circuit diagram for the illustration.

The published research results were supported by the Internal Grant Agency of University of Pardubice, the project SGFEI 2016.

\author{
David Matoušek \\ Department of Electrical Engineering \\ University of Pardubice \\ 53002 Pardubice, Czech Republic \\ david.matousek@upce.cz
}

The h-parameters define set of equations (2).

$$
\begin{aligned}
& \mathrm{V}_{\mathrm{B}}=\mathrm{h}_{11} \cdot \mathrm{I}_{\mathrm{B}}+\mathrm{h}_{12} \cdot \mathrm{V}_{\mathrm{C}} \\
& \mathrm{I}_{\mathrm{C}}=\mathrm{h}_{21} \cdot \mathrm{I}_{\mathrm{B}}+\mathrm{h}_{22} \cdot \mathrm{V}_{\mathrm{C}}
\end{aligned}
$$

By equation $V_{B}=V_{1}-V_{3}$ the first equation from the set (2) can be rewritten to following form (3).

$$
1 . \mathrm{V}_{1}-1 . \mathrm{V}_{3}-\mathrm{h}_{11} \cdot \mathrm{I}_{\mathrm{B}}-\mathrm{h}_{12} \cdot \mathrm{V}_{\mathrm{C}}=0
$$

Similarly, voltage $V_{C}$ is $V_{C}=V_{2}-V_{3}$, thus

$$
-1 . \mathrm{V}_{2}+1 . \mathrm{V}_{3}+1 . \mathrm{V}_{\mathrm{C}}=0
$$

In this case set (1) and set (2) can be written together in following form (5).

$$
\begin{aligned}
& I_{1}=g_{1} \cdot V_{1}+0 \cdot V_{2}+0 . V_{3}+1 \cdot I_{B}+0 . V_{C} \\
& I_{2}=0 . V_{1}+g_{2} \cdot V_{2}+0 . V_{3}+h_{21} \cdot I_{B}+h_{22} \cdot V_{C} \\
& I_{3}=0 . V_{1}+0 . V_{2}+g_{3} \cdot V_{3}-1 \cdot I_{B}-h_{21} \cdot I_{B}-h_{22} \cdot V_{C} \\
& 0=1 . V_{1}+0 . V_{2}-1 \cdot V_{3}-h_{11} \cdot I_{B}-h_{12} \cdot V_{C} \\
& 0=0 . V_{1}-1 \cdot V_{2}+1 \cdot V_{3}+0 . I_{B}+1 \cdot V_{C}
\end{aligned}
$$

Finally, set (5) can be rewritten to matrix form, which can be characterized by matrix (6).

\begin{tabular}{|ccc|cc|}
\hline & & & 1 & 0 \\
& $\underline{Y}$ & & $\mathrm{~h}_{21}$ & $\mathrm{~h}_{22}$ \\
& & & $-1-\mathrm{h}_{21}$ & $-\mathrm{h}_{22}$ \\
\hline 1 & 0 & -1 & $-\mathrm{h}_{11}$ & $-\mathrm{h}_{12}$ \\
0 & -1 & 1 & 0 & 1 \\
\hline
\end{tabular}

We can see that matrix (6) includes both types of parameters: y-parameters and the h-parameters, too. It means, that circuit is described by different types of parameters in one matrix. From (6) one important result may by constructed: the conversion of the parameters is not performed, now.

We can see that matrix (6) is a special case of the diakoptical method described in especially e.g. [3], [4], [5], i.e. decomposition of large networks [6], [7], [8], [9] and in many others, e.g. in SC circuits [10], $[11]$, too. 


\section{EXAMPLE}

The proposed method, which is described above, will be illustrated by next solved example. Schematic diagram of this example is shown in Fig. 2.

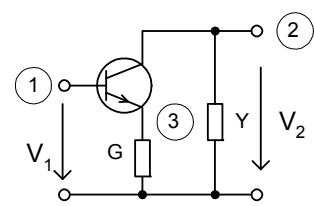

Figure 2. Circuit diagram for the example.

Matrix of this circuit based on generally matrix (6) will be in form (7).

$$
\begin{array}{|ccc|cc|}
\hline 0 & 0 & 0 & 1 & 0 \\
0 & \mathrm{Y} & 0 & \mathrm{~h}_{21} & \mathrm{~h}_{22} \\
0 & 0 & \mathrm{G} & -1-\mathrm{h}_{21} & -\mathrm{h}_{22} \\
\hline 1 & 0 & -1 & -\mathrm{h}_{11} & -\mathrm{h}_{12} \\
0 & -1 & 1 & 0 & 1 \\
\hline
\end{array}
$$

Thus voltage transfer ratio is (8).

$$
\begin{aligned}
& \frac{\mathrm{V}_{2}}{\mathrm{~V}_{1}}=\frac{\Delta_{1: 2}}{\Delta_{1: 1}}=\frac{\left|\begin{array}{cccc}
0 & 0 & \mathrm{~h}_{21} & \mathrm{~h}_{22} \\
0 & \mathrm{G} & -1-\mathrm{h}_{21} & -\mathrm{h}_{22} \\
1 & -1 & -\mathrm{h}_{11} & -\mathrm{h}_{12} \\
0 & 1 & 0 & 1
\end{array}\right|^{(-1)^{3}}}{\left|\begin{array}{cccc}
\mathrm{Y} & 0 & \mathrm{~h}_{21} & \mathrm{~h}_{22} \\
0 & \mathrm{G} & -1-\mathrm{h}_{21} & -\mathrm{h}_{22} \\
0 & -1 & -\mathrm{h}_{11} & -\mathrm{h}_{12} \\
-1 & 1 & 0 & 1
\end{array}\right|(-1)^{2}}= \\
& =\frac{-\left|\begin{array}{ccc}
0 & \mathrm{~h}_{21} & \mathrm{~h}_{22} \\
\mathrm{G} & -1-\mathrm{h}_{21} & -\mathrm{h}_{22} \\
1 & 0 & 1
\end{array}\right|(-1)^{4}}{\mathrm{Y}\left|\begin{array}{ccc}
\mathrm{G} & -1-\mathrm{h}_{21} & -\mathrm{h}_{22} \\
-1 & -\mathrm{h}_{11} & -\mathrm{h}_{12} \\
1 & 0 & 1
\end{array}\right|-\left|\begin{array}{ccc}
0 & \mathrm{~h}_{21} & \mathrm{~h}_{22} \\
\mathrm{G} & -1-\mathrm{h}_{21} & -\mathrm{h}_{22} \\
-1 & -\mathrm{h}_{11} & -\mathrm{h}_{12}
\end{array}\right|}= \\
& =\frac{-h_{22} \cdot h_{21}-h_{22} \cdot\left(-1-h_{21}\right)-G \cdot h_{21}}{Y \cdot\left[-h_{11} \cdot G+h_{12}+h_{12} \cdot h_{21}-h_{11} \cdot h_{22}-1-h_{21}\right]}= \\
& +\left[h_{21} \cdot h_{22}-G \cdot h_{11} \cdot h_{22}-h_{22}-h_{21} \cdot h_{22}+G \cdot h_{21} \cdot h_{12}\right] \cdot(-1) \\
& =\frac{h_{22}-G \cdot h_{21}}{Y \cdot h_{11} \cdot G-Y \cdot h_{12}+Y \cdot\left(h_{11} \cdot h_{22}-h_{12} \cdot h_{21}\right)+Y+Y \cdot h_{21}} \\
& +\mathrm{G} \cdot\left(\mathrm{h}_{11} \cdot \mathrm{h}_{22}-\mathrm{h}_{12} \cdot \mathrm{h}_{21}\right)+\mathrm{h}_{22}
\end{aligned}
$$

\section{COMPARISON}

The difference between two standard solving methods may be shown in the following example. For comparison the same circuit is solved by Nodal Voltage Method with conversion h-parameters to y-parameters.

In the first step, we write equations for h-parameters definition (9).

$$
\begin{aligned}
& \mathrm{V}_{\mathrm{B}}=\mathrm{h}_{11} \cdot \mathrm{I}_{\mathrm{B}}+\mathrm{h}_{12} \cdot \mathrm{V}_{\mathrm{C}} \\
& \mathrm{I}_{\mathrm{C}}=\mathrm{h}_{21} \cdot \mathrm{I}_{\mathrm{B}}+\mathrm{h}_{22} \cdot \mathrm{V}_{\mathrm{C}}
\end{aligned}
$$

In the second step, we derive h-parameters by $\mathrm{V}_{\mathrm{CE}}=0$ or $\mathrm{I}_{\mathrm{B}}=0$ condition (10).

$$
\begin{array}{cc}
\mathrm{h}_{11}=\left.\frac{\mathrm{V}_{\mathrm{B}}}{\mathrm{I}_{\mathrm{B}}}\right|_{\mathrm{V}_{\mathrm{C}}=0} \quad \mathrm{~h}_{21}=\left.\frac{\mathrm{I}_{\mathrm{C}}}{\mathrm{I}_{\mathrm{B}}}\right|_{\mathrm{V}_{\mathrm{C}}=0} \\
\mathrm{~h}_{12}=\left.\frac{\mathrm{V}_{\mathrm{B}}}{\mathrm{V}_{\mathrm{C}}}\right|_{\mathrm{I}_{\mathrm{B}}=0} \quad \mathrm{~h}_{22}=\left.\frac{\mathrm{I}_{\mathrm{C}}}{\mathrm{V}_{\mathrm{C}}}\right|_{\mathrm{I}_{\mathrm{B}}=0}
\end{array}
$$

In the third step, we write y-parameters of BJT (11).

$$
\begin{aligned}
& I_{B}=y_{11} \cdot V_{B}+y_{12} \cdot V_{C} \\
& I_{C}=y_{21} \cdot V_{B}+y_{22} \cdot V_{C}
\end{aligned}
$$

In the fourth step, we convert h-parameters to y-parameters (12).

$$
\begin{gathered}
\mathrm{y}_{11}=\left.\frac{\mathrm{I}_{\mathrm{B}}}{\mathrm{V}_{\mathrm{B}}}\right|_{\mathrm{V}_{\mathrm{C}}=0}=\frac{1}{\left.\frac{\mathrm{V}_{\mathrm{B}}}{\mathrm{I}_{\mathrm{B}}}\right|_{\mathrm{V}_{\mathrm{C}}=0}}=\frac{1}{\mathrm{~h}_{11}} \\
\mathrm{y}_{21}=\left.\frac{\mathrm{I}_{\mathrm{C}}}{\mathrm{V}_{\mathrm{B}}}\right|_{\mathrm{V}_{\mathrm{C}}=0}=\left.\frac{\mathrm{I}_{\mathrm{B}}}{\mathrm{I}_{\mathrm{B}}} \frac{\mathrm{I}_{\mathrm{C}}}{\mathrm{V}_{\mathrm{B}}}\right|_{\mathrm{V}_{\mathrm{C}}=0}=\left.\left.\frac{\mathrm{I}_{\mathrm{C}}}{\mathrm{I}_{\mathrm{B}}}\right|_{\mathrm{V}_{\mathrm{C}}=0} \cdot \frac{\mathrm{I}_{\mathrm{B}}}{\mathrm{V}_{\mathrm{B}}}\right|_{\mathrm{V}_{\mathrm{C}}=0}=\mathrm{h}_{21} \frac{1}{\left.\frac{\mathrm{V}_{\mathrm{B}}}{\mathrm{I}_{\mathrm{B}}}\right|_{\mathrm{V}_{\mathrm{C}}=0}}=\frac{\mathrm{h}_{21}}{\mathrm{~h}_{11}} \\
\mathrm{y}_{22}=\left.\frac{\mathrm{I}_{\mathrm{C}}}{\mathrm{V}_{\mathrm{C}}}\right|_{\mathrm{V}_{\mathrm{B}}=0}=\mathrm{h}_{22}-\mathrm{h}_{21} \cdot \frac{\mathrm{h}_{12}}{\mathrm{~h}_{11}}=\frac{\mathrm{h}_{11} \cdot \mathrm{h}_{22}-\mathrm{h}_{21} \cdot \mathrm{h}_{12}}{\mathrm{~h}_{11}}
\end{gathered}
$$

We can see, that presented conversion of h-parameter to y-parameters is more complicated than the assembly diakoptical transformation equations.

Finally, in the last step, the admittance matrix $\mathrm{Y}$ is in following form (13).

\begin{tabular}{|c|c|c|}
\hline$\frac{1}{\mathrm{~h}_{11}}$ & $-\frac{\mathrm{h}_{12}}{\mathrm{~h}_{11}}$ & $\frac{\mathrm{h}_{12}}{\mathrm{~h}_{11}}-\frac{1}{\mathrm{~h}_{11}}$ \\
\hline$\frac{\mathrm{h}_{21}}{\mathrm{~h}_{11}}$ & $\frac{\mathrm{h}_{11} \cdot \mathrm{h}_{22}-\mathrm{h}_{12} \cdot \mathrm{h}_{21}}{\mathrm{~h}_{11}}+\mathrm{Y}$ & $-\frac{\mathrm{h}_{21}}{\mathrm{~h}_{11}}-\frac{\mathrm{h}_{11} \cdot \mathrm{h}_{22}-\mathrm{h}_{12} \cdot \mathrm{h}_{21}}{\mathrm{~h}_{11}}$ \\
\hline$-\frac{1}{\mathrm{~h}_{11}}-\frac{\mathrm{h}_{21}}{\mathrm{~h}_{11}}$ & $\frac{\mathrm{h}_{12}}{\mathrm{~h}_{11}}-\frac{\mathrm{h}_{11} \cdot \mathrm{h}_{22}-\mathrm{h}_{12} \cdot \mathrm{h}_{21}}{\mathrm{~h}_{11}}$ & $\frac{\mathrm{h}_{12}}{\mathrm{~h}_{11}}+\frac{\mathrm{h}_{11} \cdot \mathrm{h}_{22}-\mathrm{h}_{12} \cdot \mathrm{h}_{21}}{\mathrm{~h}_{11}}+\frac{1}{\mathrm{~h}_{11}}+\frac{\mathrm{h}_{21}}{\mathrm{~h}_{11}}+\mathrm{G}$ \\
\hline
\end{tabular}


Calculation of the voltage transfer ratio from matrix (13) is more complex and not transparent with comparison with (8), too. This situation is documented by equation (14), when we use $\Delta h=h_{11} \cdot h_{22}-h_{21} \cdot h_{12}$.

$$
\begin{aligned}
& \frac{\mathrm{V}_{2}}{\mathrm{~V}_{1}}=\frac{\Delta_{1: 2}}{\Delta_{1: 1}}=
\end{aligned}
$$

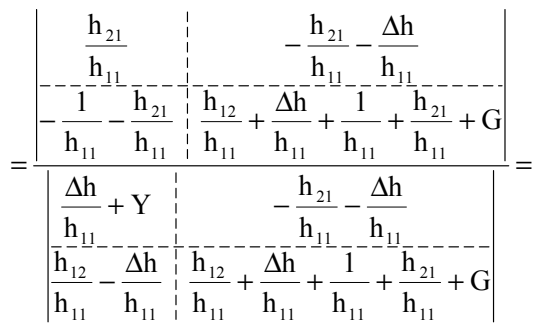

$$
\begin{aligned}
& =\frac{\frac{\mathrm{h}_{21}}{\mathrm{~h}_{11}}\left(\frac{\mathrm{h}_{12}+\Delta \mathrm{h}+1+\mathrm{h}_{21}}{\mathrm{~h}_{11}}+\mathrm{G}\right)-\left(-\frac{1+\mathrm{h}_{21}}{\mathrm{~h}_{11}}\right)\left(-\frac{\mathrm{h}_{21}+\Delta \mathrm{h}}{\mathrm{h}_{11}}\right)}{\left(\frac{\Delta \mathrm{h}}{\mathrm{h}_{11}}+\mathrm{Y}\right)\left(\frac{\mathrm{h}_{12}+\Delta \mathrm{h}+1+\mathrm{h}_{21}}{\mathrm{~h}_{11}}+\mathrm{G}\right)-\left(\frac{\mathrm{h}_{12}-\Delta \mathrm{h}}{\mathrm{h}_{11}}\right)\left(-\frac{\mathrm{h}_{21}+\Delta \mathrm{h}}{\mathrm{h}_{11}}\right)}= \\
& \ldots \\
& =\frac{h_{22}-G \cdot h_{21}}{Y \cdot h_{11} \cdot G-Y \cdot h_{12}+Y \cdot \Delta h+Y+Y \cdot h_{21}+G \cdot \Delta h+h_{22}}
\end{aligned}
$$

\section{ANOTHER VARIANT COMPILATION OF EQUATIONS}

The described method for compilation of the matrix is not one way of circuit solving only, of course. Let's use the principles described in [12]. If we substitute the equation (15) to the second equation from set (2), then the fourth equation from set (5) will be in following form (16).

$$
\begin{gathered}
\mathrm{V}_{\mathrm{C}}=\mathrm{V}_{2}-\mathrm{V}_{3} \\
0=1 \cdot \mathrm{V}_{1}+0 \cdot \mathrm{V}_{2}-1 \cdot \mathrm{V}_{3}-\mathrm{h}_{11} \cdot \mathrm{I}_{\mathrm{B}}-\mathrm{h}_{12} \cdot \mathrm{V}_{\mathrm{C}}
\end{gathered}
$$

In this case, the set of equations can be characterized by new matrix (17).

$$
\begin{array}{|ccc|cc|}
\hline & & & 1 & 0 \\
& \underline{Y} & & \mathrm{~h}_{21} & \mathrm{~h}_{22} \\
& & & -1-\mathrm{h}_{21} & -\mathrm{h}_{22} \\
\hline 1 & -\mathrm{h}_{12} & -1+\mathrm{h}_{12} & -\mathrm{h}_{11} & 0 \\
0 & -1 & 1 & 0 & 1 \\
\hline
\end{array}
$$

Now we consider the circuit from Fig. 2 again. Matrix of this circuit based on generally matrix (17) will be in form (18).

\begin{tabular}{|ccc|cc|}
\hline 0 & 0 & 0 & 1 & 0 \\
0 & $\mathrm{Y}$ & 0 & $\mathrm{~h}_{21}$ & $\mathrm{~h}_{22}$ \\
0 & 0 & $\mathrm{G}$ & $-1-\mathrm{h}_{21}$ & $-\mathrm{h}_{22}$ \\
\hline 1 & $-\mathrm{h}_{12}$ & $-1+\mathrm{h}_{12}$ & $-\mathrm{h}_{11}$ & 0 \\
0 & -1 & 1 & 0 & 1 \\
\hline
\end{tabular}

And the voltage transfer ratio is calculated by equation (19), now.

$$
\begin{aligned}
& \frac{\mathrm{V}_{2}}{\mathrm{~V}_{1}}=\frac{\Delta_{1: 2}}{\Delta_{1: 1}}=\frac{\left|\begin{array}{cccc}
0 & 0 & \mathrm{~h}_{21} & \mathrm{~h}_{22} \\
0 & \mathrm{G} & -1-\mathrm{h}_{21} & -\mathrm{h}_{22} \\
1 & -1+\mathrm{h}_{12} & -\mathrm{h}_{11} & 0 \\
0 & 1 & 0 & 1
\end{array}\right|(-1)^{3}}{\left|\begin{array}{cccc}
\mathrm{Y} & 0 & \mathrm{~h}_{21} & \mathrm{~h}_{22} \\
0 & \mathrm{G} & -1-\mathrm{h}_{21} & -\mathrm{h}_{22} \\
0 & -1+\mathrm{h}_{12} & -\mathrm{h}_{11} & 0 \\
-\mathrm{h}_{12} & 1 & 0 & 1
\end{array}\right|(-1)^{2}}=
\end{aligned}
$$

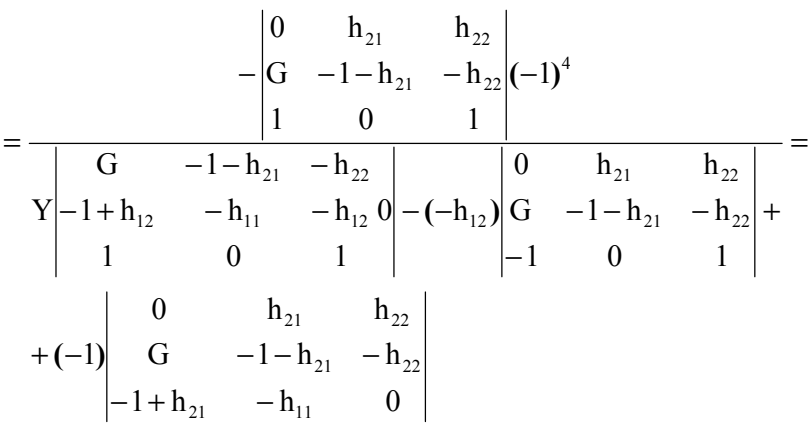

Comparison of equations (14) and (19) shows clearly, that the appeared denominator has three members while in the previous case there are two members only. Thus calculation using the relationship (16) is more complicated. This is due to the fact that in the matrix appearing more non-zeroed elements or elements outside the main diagonal. Described fact is evident from the comparison of the lower two submatrices in general matrices (6), (17) and also from matrices of a particular circuit (7), (18) of Fig. 2.

\section{CONCLUSION}

Analysis of the electric circuits is necessary not only for computing of circuit properties but also for understanding their principles. Computer methods are a powerful tool for symbolic analysis of circuit parameters. But it is advantageous to have a tool capable of clear and simple symbolic analysis, too.

In this article was described the procedure for obtaining a matrix assembly algorithm when the Nodal Voltage Method is used for solving circuit with BJTs which are described by their h-parameters.

Described method is especially determined for solution smaller large circuits "by hand", where solution "by hand" is simpler than the (often very complicated) dialogue with the computer program. Therefore a computer application is not presented. Described method requires neither intermediate rather laborious conversion h-parameters to y-parameters.

There are described two variants of compilation transformation equations for diakoptical method. Comparison demonstrates this process that is based on a less laborious calculation. 


\section{REFERENCES}

[1] J. Vlach, K. Singhal, Computer Methods for Circuit Analysis and Design, $2^{\text {nd }}$ ed. Van Nostrand Reynhold, New York, 2003, pp.68-73.

[2] J. Pospíšil, T. Dostál, "Electronic networks and systems I." Printed lectures, FE BUT Brno, 1991 (in Czech).

[3] G. N. Stenbakken, J. A. Starzyk, "Diakoptic and large change sensitivity snalysis," IEEE Proceedings-G, vol.139, No.1, pp.114-118, February 1992.

[4] A. I. Rybin, J. K. Trochimenko, M. V. Radionova, "Analysis of irregular networks using diakoptic modification of admittance matrix inversion", Radioengineering vol.2, No.4, pp.9-12, December 1993.

[5] O. Yevsyeyveva, "Diakoptical Approach in Telecommunication Engineering“" TCSET'2010, pp.29, LvivSlavske, Ukraine, February 23-27, 2010.

[6] J. A. Staryzk, A. Koncyzkowska, "Flowgraph Analysis of Large Electronic Networks" IEE Transactions on Circuits and Systems, vol.33, No.3, pp.302-315, March 1986.
[7] J. A. Staryzk, "Topological Analysis and Diagnostics of Analog Circuits", Wydawnictwo Politechniky Slaskej, Gliwice, 2007, pp.17-75.

[8] J. Brzobohatý, J. Pospíšil, "General transformation attribute of linear circuits", Electronics Horizon, vol.48, No.6, pp.1-10, July 1987.

[9] A. I. Rybin, T. Dostál, "Nodal Analysis of Circuits Containing Current Conveyors" ," Radioengineering vol.10 No.3, pp.1-4, September 2001

[10] T. Dostál, A. I. Rybin, J. K. Trochimenko, "Calculation of Poles of Network Functions Diakoptical Modification of Complex Circuits“, Electronics Horizon, special issue, pp.4547, 1994.

[11] K. Gorshkov, V. V. Filaretov, S. A. Kurganov, Parameters Extraction Technique for Optimal Network Functions of SC Circuits. International Siberian Conference on Control and Communications (SIBCON), May, 2015, pp.215-220.

[12] J. Čajka, J. Kvasil, “Theory of linear circuits", SNTL/ALFA Praha/Bratislava, 1979, pp.41-137. 